\title{
Ion transfer mechanisms in Mrp-type antiporters from high resolution cryoEM and molecular dynamics simulations
}

\author{
Yongchan Lee ${ }^{1,2,+}$, Outi Haapanen ${ }^{3,+}$, Anton Altmeyer ${ }^{4,5}$, Werner Kühlbrandt ${ }^{1}$, Vivek \\ Sharma ${ }^{3,6, *}$ and Volker Zickermann ${ }^{4,5, *}$
}
${ }^{1}$ Department of Structural Biology, Max Planck Institute of Biophysics, 60438 Frankfurt am Main, Germany.
${ }^{2}$ Present address: Graduate School of Medical Life Science, Yokohama City University, 230- 0045 Kanagawa, Japan.

${ }^{3}$ Department of Physics, University of Helsinki, 00014 Helsinki, Finland.

${ }^{4}$ Institute of Biochemistry II, University Hospital, Goethe University, 60438 Frankfurt am Main, Germany.

${ }^{5}$ Centre for Biomolecular Magnetic Resonance, Institute for Biophysical Chemistry, Goethe University, 60438 Frankfurt am Main, Germany.

${ }^{6}$ HiLIFE Institute of Biotechnology, University of Helsinki, 00014 Helsinki, Finland.

${ }^{\dagger}$ contributed equally

* correspondence

vivek.sharma@helsinki.fi

zickermann@med.uni-frankfurt.de

\begin{abstract}
Multiple resistance and $\mathrm{pH}$ adaptation (Mrp) cation/proton antiporters are essential for growth of a variety of halophilic and alkaliphilic bacteria under stress conditions. Mrp-type antiporters are closely related to the membrane domain of respiratory complex I. We determined the structure of the Mrp antiporter from Bacillus pseudofirmus by electron cryo-microscopy at $2.2 \AA$ resolution. The structure resolves more than $99 \%$ of the sidechains of the seven membrane subunits MrpA to MrpG plus 360 water molecules, including 70 in putative ion translocation pathways. Molecular dynamics simulations based on the highresolution structure revealed details of the antiport mechanism. We find that switching the position of a histidine residue between three hydrated pathways in the MrpA subunit is critical for proton transfer that drives gated transmembrane sodium translocation. Several lines of evidence indicate that the same histidine-switch mechanism operates in respiratory complex I.
\end{abstract}


bioRxiv preprint doi: https://doi.org/10.1101/2022.01 24.477492; this version posted January 24, 2022. The copyright holder for this preprint (which was not certified by peer review) is the author/funder, who has granted bioRxiv a license to display the preprint in perpetuity. It is made available under aCC-BY-NC-ND 4.0 International license.

\section{Introduction}

Sodium/proton antiporters are essential to maintain an intracellular homeostasis of $\mathrm{pH}$ and sodium concentration (1-3). Cation/proton antiporters (CPAs) of the CPA1 and CPA2 families, such as NhaA and NHE, are structurally and functionally fully characterized $(2,4)$. The CPA3 family comprises the much larger Mrp (multiple resistance and $\mathrm{pH}$ adaptation) type antiporters which have received much attention because of their evolutionary relationship to redox-driven ion pumps of the complex I superfamily (5-9). Energyconverting complexes of the complex I type all combine different sets of Mrp antiporter-like membrane subunits with a membrane-anchored hydrophilic redox unit.

The Mrp operon was first identified in a Bacillus species $(10,11)$. Since then, Mrp-type antiporters have been found in many halophilic and alkaliphilic bacteria (12). They can be divided into two large groups. Group I Mrp antiporters consist of seven proteins (MrpAMrpG). In group II, MrpA and B are fused while all other proteins are single polypeptides (MrpA' and MrpC-MrpG). The hetero-oligomeric complexes have been isolated in monomeric and dimeric form (13-15). CryoEM structures of two Mrp antiporters have been determined recently, one from Dietzia sp. (15), the other from Anoxybacillus flavithermus (14). Both differ considerably from those of the other two CPA families.

The largest subunit MrpA consists of two domains connected by a helix on the cytoplasmic side, sometimes referred to as the lateral helix. The overall structure of the $\mathrm{N}$-terminal core domain is similar to that of the MrpD subunit and the fold is also conserved in the respiratory complex I subunits ND5, ND4 and ND2 (14-16). The C-terminal domain of MrpA resembles the ND6 subunit of complex I (17). MrpC is related to complex I subunit ND4L (17). More recently, it was shown that also subunits of membrane bound hydrogenase $(\mathrm{MBH})(18)$ and elemental sulfur reductase (MBS) (19) are clearly related to subunits of the antiporter.

Despite the surge in structural information, the ion translocation pathways in the complex I superfamily and in Mrp-type antiporters are debated. For the antiporter, several mutually exclusive models have been proposed $(5,14,15,18)$. Based on molecular simulations, a recently published study (20) suggests transfer pathways for sodium in $\mathrm{MBH}$ that differ significantly from the original interpretations of the structure (18). With respect to complex 
I, we and others have recently questioned the previous consensus model that assumes four complete and self-contained proton pumping paths $(21,22)$. A molecular understanding of the Mrp antiporter is therefore also of great importance for fundamental questions relating to complex I function and biological energy conversion. In this work, we determined the cryoEM structure of the Mrp antiporter from Bacillus pseudofirmus at $2.2 \AA$ resolution. The antiporter complex from this organism is well studied biochemically and by site-directed mutagenesis $(13,23-25)$. Our high-resolution structure enabled us to identify ion translocation pathways by modelling water molecules in hydrophobic transmembrane regions. Our maps give clear indications for conformational changes in key regions of the structure with far-reaching mechanistic implications. Molecular dynamics (MD) simulations performed on the basis of the high-resolution cryoEM structure provide the atomistic determinants underlying observed conformational changes. In addition, MD simulations reveal putative sodium ion binding sites and how sodium ion translocation may depend on protonation and conformational states of the antiporter.

\section{Results}

\section{High resolution structure}

We expressed recombinant His-tagged B. pseudofirmus Mrp antiporter complex in E. coli and purified it by affinity chromatography in lauryl maltose neopentyl glycol (LMNG). Electron cryo-microscopy (cryoEM) of the purified complex revealed a dimer of heteroheptamers, consistent with previous blue-native PAGE analyses of this particular complex (13). Single-particle data analysis showed varying angles between the two protomers, limiting the map resolution to around $3 \AA$ even after extensive 3D classification. Therefore, we resorted to symmetry expansion and focused 3D refinement (Fig. S1, Table S1), in an approach similar to that taken by Steiner and Sazanov (14) for the ortholog Mrp antiporter from $A$. flavithermus. The final 3D reconstruction of the protomer yielded a 2.2- $\AA$ map, allowing us to build a model including 1957 amino acid residues, three phospholipids and 360 water molecules. The map did not show significant differences for the two protomers in the dimeric complex, and therefore we can confine ourselves to a discussion of the monomeric structure. 

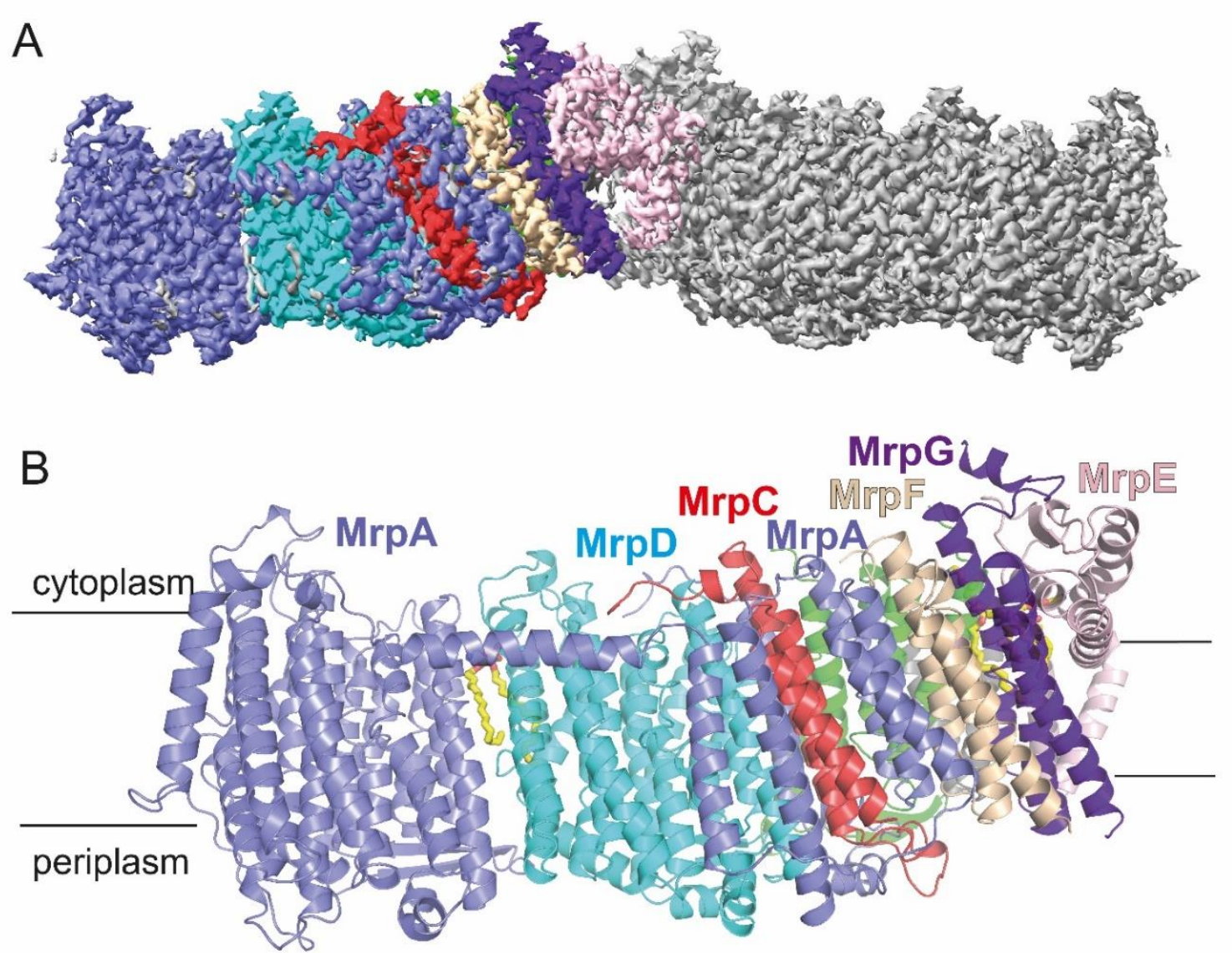

C

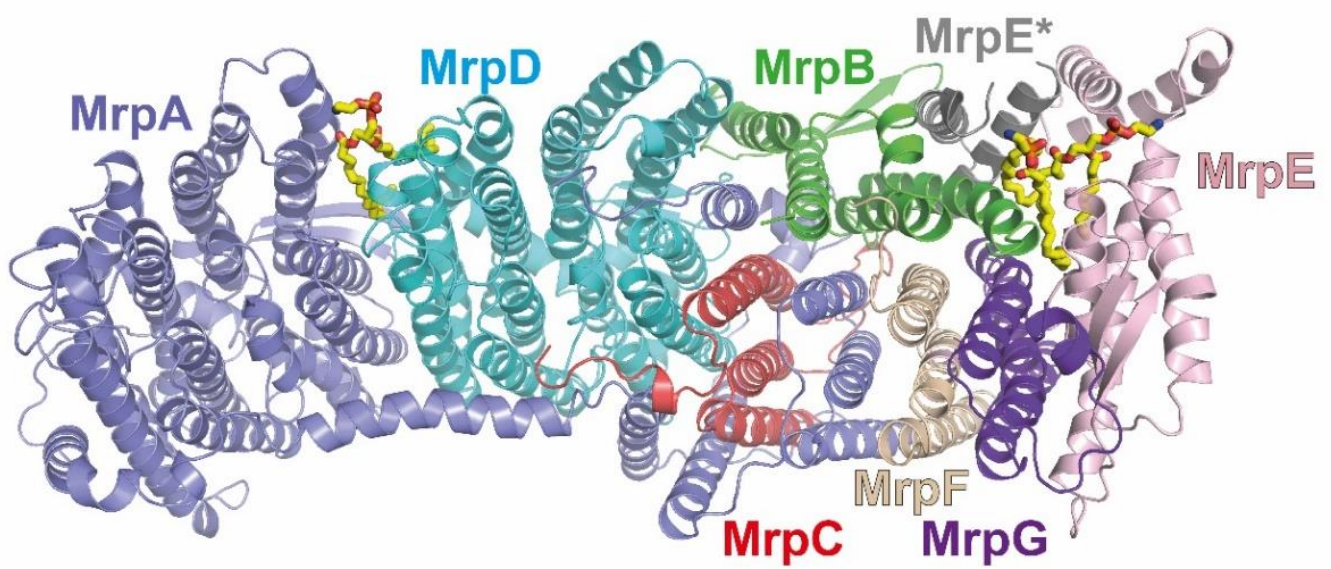

Fig. 1. Overall structure of B. pseudofirmus $\mathrm{Mrp} \mathrm{Na} \mathbf{H}^{+} / \mathrm{H}^{+}$antiporter. (A) CryoEM density for a dimer, (B) side view with and (C) top view of a protomer comprising the seven subunits MrpA - MrpG and a partial model of MrpE from an adjacent monomer (MrpE*). In (B) approximate membrane boundaries are shown.

Each protomer of the dimeric complex has a mass of $213 \mathrm{kDa}$ and comprises seven subunits that are designated MrpA-G (Fig. 1). The fold of the N-terminal domain of MrpA with 14 
trans-membrane helices (TMH1-14) is very similar to that of the neighboring MrpD subunit. In MrpA, this core structure is extended by the so-called lateral helix that runs along the cytoplasmic membrane surface and is anchored by a TMH on the surface of the MrpD subunit. Each of the small subunits MrpC, MrpF and MrpG consists of a linear arrangement of three TMH each. The C-terminal domain of MrpA surrounds MrpC in a semicircle and contributes a layer of TMHs between MrpC and MrpF. The successive arrangement of MrpC, the C-terminal MrpA domain, MrpF and MrpG is lined by the four TMHs of the MrpB subunit. MrpE completes the structure and consists of two remarkably short TMHs and a cytoplasmic domain. MrpE forms contacts with MrpE and MrpB from the other protomer. The presence of two short TMHs in MrpE might cause a thinning of the membrane at the dimer interface as suggested for the Mrp antiporter of $A$. flavithermus (14).

The similarity of subunits of the Mrp antiporter with subunits of complex I and members of the complex I superfamily $(14-17,26)$ is summarized in Table S2. Note that the conservation of amino acid residues thought to be functionally significant is extensive but not complete (Fig. S3, Fig. S4).

The overall structure of our Mrp antiporter from B. pseudofirmus agrees well with the recently published $2.98 \AA$ A structure of the antiporter dimer from A. flavithermus (14) (Fig. S5), but there are obvious differences from the $3.0 \AA$ structure of the monomeric Mrp antiporter from Dietzia sp. (15), which belongs to group 2. In the Dietzia antiporter, MrpA and MrpB are fused and the dimerization domain in MrpE is absent. All three structures show slight differences in position and inclination of the TMHs of MrpF and MrpG. Our structure is of considerably higher resolution, which enabled us to build a reliable model of protein bound water molecules (Fig. 2, Fig. S6). Furthermore, our map provides evidence of conformational changes that are most likely central to the catalytic cycle of the antiporter mechanism (see below).

\section{Alternative conformations indicate dynamic regions in the structure}

During model building we noticed clear alternative sidechain positions $A$ and $B$ (called alternate locations or altloc in the pdb) for several residues and conformational variations for short sequence stretches (Fig. 2, Fig. S2, Table S4). In MrpA, the adjacent residues Glu409 ${ }^{\mathrm{MrpA}}$ and Lys408 ${ }^{\mathrm{MrpA}}$ in $\mathrm{TMH} 12$ at the putative periplasmic proton entry site show 
alternative side chain positions (Fig. S6). Corresponding residues, based on MD simulations of bacterial complex I, have been suggested to undergo conformation-dependent proton transfer reactions (27). Furthermore, we observed a pronounced structural rearrangement in a highly conserved sequence stretch close to the N-terminal end of TMH8 (positions 246 to 252 ) resulting in alternative positions of several residues including strictly conserved His248 ${ }^{\mathrm{MrpA}}$ (Fig. 2C, D; Fig. S2, supplementary movie S1). In position A, the sidechain of His248 points towards Thr306 ${ }^{\mathrm{MrpA}}$ (Fig. 2C, D). In position B, it is oriented towards Ser146 MrpA. This group of three residues (His248, Thr306, Ser146) is strictly conserved in MrpA and complex I subunit ND5 (Fig. S3) and the homologous histidine has been shown to undergo protonation-state dependent conformational changes in complex I MD simulations (27). Note that also the N-terminal sequence of TMH8 in MrpA (position 240 to 244) is highly conserved and its two proline residues (position 240 and 242) may form a rigid structure that provides a counter bearing for conformational changes in the adjacent flexible segment (Fig. 2C). In addition, the movement of Leu247 ${ }^{\mathrm{MrpA}}$ in TMH8 is associated with a rearrangement of Phe119 ${ }^{\mathrm{MrpA}}$ in TMH4 (Fig. 2D).

In MrpD, we modelled the sidechain of residue Lys219 in two alternative positions (Fig. 2, Fig. S2, Fig. S6). This residue is part of the strictly conserved Lys/Glu pair of this subunit that is discussed to play a mechanistic key role in Mrp antiporters and complex I (reviewed in (8)).

In the C-terminal domain of MrpA, we modelled Glu687 ${ }^{\mathrm{MrpA}}$ in $\mathrm{TMH} 19$ with two alternative positions of its sidechain (Fig. 2, Fig S2). Interestingly, also densities for hydrophobic residues including Phe $41^{\mathrm{MrpB}}$ of $\mathrm{MrpB}$ showed strong indications for alternative conformations (Fig. 2, Fig. S2). Phe41 ${ }^{\mathrm{MrpB}}$ is part of a strictly conserved Pro-Gly-Gly-Gly-Phe sequence stretch in MrpB (Fig. S3). Mutagenesis data indicate an important function for Glu687 ${ }^{\mathrm{MrpA}}$ and $\mathrm{Phe} 41^{\mathrm{MrpB}}(15,23,25)$ and the conformational variations of both residues may be related to sodium translocation (see below). 

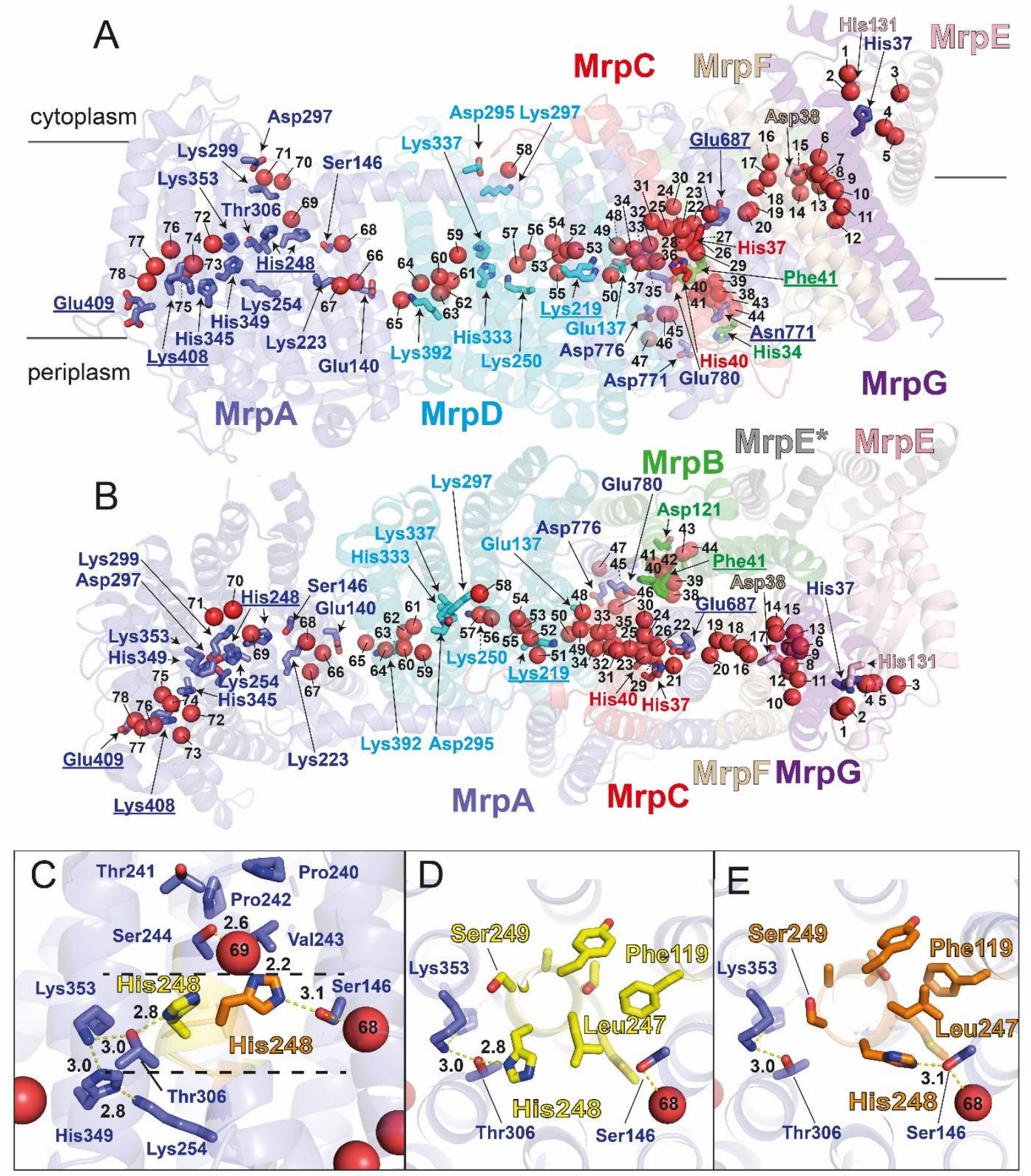

Fig. 2. Alternative conformations, water molecules and functionally important residues.

(A) Side view and (B) top view of antiporter complex (colors compare Fig. 1) with 78 protein bound water molecules (red spheres) in putative ion translocation pathways. Selected residues are shown in stick representation and labels for residues modelled with alternative conformations are underlined. (C) Side view of the protein structure surrounding His $248^{\mathrm{MrpA}}$. In the $A$ conformation (yellow) His $248^{\mathrm{MrpA}}$ interacts with $\mathrm{Thr} 306^{\mathrm{MrpA}}$, in the $B$ conformation (orange) it engages in a hydrogen bond with Ser146 ${ }^{\mathrm{MrpA}}$ (see supplementary movie S1). (D, E) Top view at the level of the mobile segment of TMH8 showing $A$ and $B$ conformation (colors as in C). Movement of Leu247 is associated with a shift in sidechain position of Phe119 ${ }^{\mathrm{MrpA}}$ in TMH4. 
bioRxiv preprint doi: https://doi.org/10.1101/2022.01 24.477492; this version posted January 24, 2022. The copyright holder for this preprint (which was not certified by peer review) is the author/funder, who has granted bioRxiv a license to display the preprint in perpetuity. It is made available under aCC-BY-NC-ND 4.0 International license.

\section{Water molecules, buried charges and polar residues identify ion translocation pathways}

Most of the 360 modelled water molecules are located on the hydrophilic protein surfaces exposed to the cytoplasm or periplasm as expected, but about 70 water molecules are found in the interior of the transmembrane region (Fig. 2, Fig. S6). The internal waters are arranged along a central hydrophilic axis that extends over the entire length of the antiporter complex. Our detailed analysis (supplementary text) shows that protonatable and polar residues are equally important for coordinating water molecules in the complex interior, and thus for the formation of ion translocation pathways.

In MrpA, His248 ${ }^{\mathrm{MrpA}}$ is located at the junction of three hydrated pathways. In the $A$ conformation, there is a connection via Thr306 ${ }^{\mathrm{MrpA}}$ with a remarkable arrangement of the three strictly conserved residues His349 MrpA, Lys254 ${ }^{\mathrm{MrpA}}$ and Lys353 ${ }^{\mathrm{MrpA}}$ (Fig. 2). From there, water molecules W74 - W78 form a connection to residues Lys408 MrpA $/$ Glu409 MrpA at the presumed proton entry site on the periplasmic side. In the $B$ conformation, there is a connection via Ser146 ${ }^{\mathrm{MrpA}}$ to a water chain (W66 - W68) that leads to the strictly conserved Glu140 MrpA/Lys223 ${ }^{\mathrm{MrpA}}$ pair of MrpA. A connection to the cytosolic side is formed by polar residues, including strictly conserved Ser244 ${ }^{\mathrm{MrpA}}$, and associated water molecules W69W71 (Fig. 2).

In MrpD, Lys392 ${ }^{\mathrm{MrpD}}$ in TMH12 is in the same position as Lys408 ${ }^{\mathrm{MrpA}}$ in MrpA and the arrangement of the three residues Lys $250^{\mathrm{MrpD}}$, His $333^{\mathrm{MrpD}}$ and Lys $337^{\mathrm{MrpD}}$ corresponds to the Lys-His-Lys triad in MrpA described above (for conservation see supplemental text and Fig. S7). As in MrpA, there is a connection of water molecules (W59 - W64) between the TMH12 lysine and the Lys-His-Lys triad in MrpD. In contrast to MrpA, there is no obvious connection by water molecules to the periplasm and there is no residue corresponding to the mobile His248 ${ }^{\mathrm{MrpA}}$ in TMH8 of MrpD. A connection from the central Lys $250^{\mathrm{MrpD}}$ to the cytoplasmic side is expected but there are no water molecules in this region of the structure. This might indicate that the pathway to the cytoplasm is blocked by the highly conserved Phe341 ${ }^{\mathrm{MrpD}}$ in TMH11 (Fig. S3) as recently described for complex I (22). An overlay of ND2, ND4 and MrpD shows that the position of this residue agrees well with the notion of a closed proton transfer path in the ND4 subunit (Fig. S4). 
TMH2 and TMH3 of MrpC are located at the center of a highly hydrated region. Waters W32 to W37 sit between MrpC and the strictly conserved Glu137MrpD/Lys219MrpD pair of MrpD; on the other side, W21 - W31 connect MrpC with the strictly conserved Glu687 ${ }^{\mathrm{MrpA}}$ in TMH19 of the C-terminal domain of MrpA. From here, a string of water molecules W16 W20 continues to a hydrated region close to Asp38 ${ }^{\mathrm{MrpF}}$. In agreement with a previous suggestion (14), the pronounced hydration in the vicinity of MrpC suggests a role in sodium transfer, but the water molecules found in the structure did not provide clear information about the connection pathways to the periplasmic or cytosolic side. We have therefore investigated this question further and conducted an analysis of the structure with Caver and MD simulation approaches.

\section{Cavities in the structure suggest sodium transport pathways}

We used the software tool Caver (28) to identify potential sodium transfer pathways with connections to the periplasmic or cytoplasmic side (Fig. 3). Since residues corresponding to Glu687 ${ }^{\mathrm{MrpA}}$ in TMH19 are potentially critical for sodium translocation in Mrp antiporters (14, 29) and in $\mathrm{MBH}(20)$, we used this residue as starting point for a search with a minimal probe radius of $1.2 \AA$. We found a connection to the cytosolic side close to $\operatorname{Arg} 27^{\mathrm{MrpC}}$ and Glu707 ${ }^{\mathrm{MrpA}}$ but the interior of this cavity at the interface of $\mathrm{MrpC}$ and the $\mathrm{C}$-terminal domain of MrpA is predominantly lined by hydrophobic residues (Fig. 3A).

A more extensive system of cavities was found when the probe radius was reduced to $0.8 \AA$. This revealed a hydrophilic tunnel containing water molecules W16 - 20 leading from Glu687 ${ }^{\mathrm{MrpA}}$ to Asp38 ${ }^{\mathrm{MrpF}}$. However, access for sodium to Asp38 ${ }^{\mathrm{MrpF}}$ from the cytoplasmic side is not obvious in the Caver analysis.

The two water-filled cavities at the $\mathrm{MrpA} / \mathrm{MrpC} / \mathrm{MrpD}$ interface open a putative pathway for sodium from Glu687 ${ }^{\mathrm{MrpA}}$ to the Lys/Glu pair of MrpD (Fig. 2, Fig.S6). For the Mrp antiporter of $A$. flavithermus (14) a connection from the MrpD glutamate to the periplasm was proposed along the interface of $\mathrm{MrpA}(\mathrm{TMH} 21) / \mathrm{MrpC}(\mathrm{TMH} 3) / \mathrm{MrpD}(\mathrm{TMH} 5)$. In the $B$. pseudofirmus structure Caver does not detect this pathway with reasonable probe radii, as it is blocked by hydrophobic sidechains. 


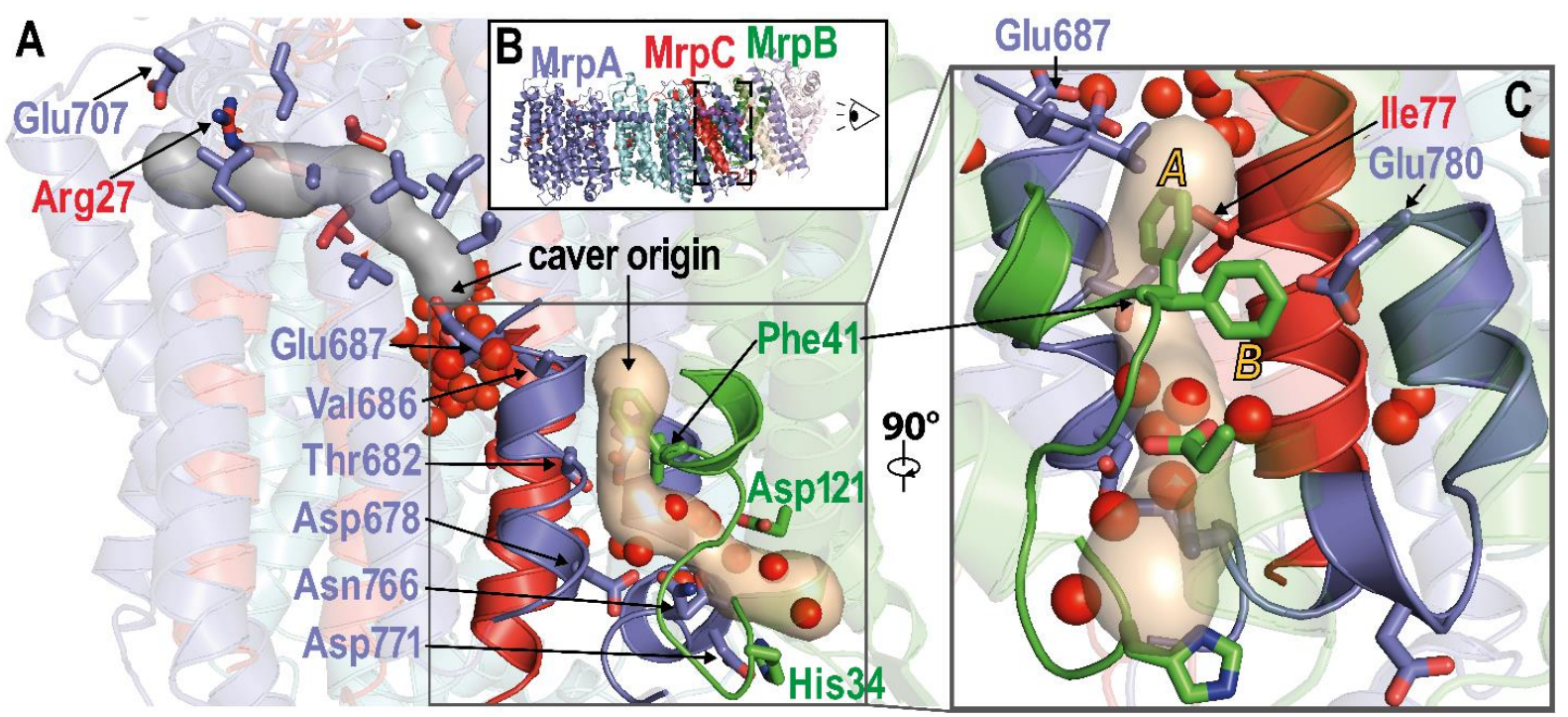

Fig. 3. Putative sodium transfer pathways. (A) Slice view on the C-terminal domain of MrpA as indicated in (B). Tunnels identified with a minimal probe radius of $1.2 \AA$ originating at Glu687 MrpA (grey) and above Phe41 ${ }^{\mathrm{MrpB}}$ (wheat) are shown in surface view at $20 \%$ transparency. The grey tunnel starts at the central water axis around W18 to W31 and terminates at the cytoplasmic side close to Glu707 $\mathrm{MrpA}$ and $\operatorname{Arg} 27^{\mathrm{MrpC}}$. The tunnel is lined by several hydrophobic and poorly conserved residues (lle650 MrpA, Ala656 MrpA, Val657 ${ }^{\mathrm{MrpA}}$, Val660 ${ }^{\text {MrpA }}$, Val691 ${ }^{\text {MrpA }}$, Leu694 ${ }^{\mathrm{MrpA}}$, Leu704 ${ }^{\mathrm{MrpA}}$, Leu26 ${ }^{\mathrm{MrpC}}$ and Val30 ${ }^{\mathrm{MrpC}}$ ). The wheat-colored tunnel originates above Phe $41^{\mathrm{MrpB}}$ at the level of Glu68 $7^{\mathrm{MrpA}}$ but is separated from this residue by the hydrophobic residues Val686 ${ }^{\mathrm{MrpA}}$ and Ile $77^{\mathrm{MrpC}}$. The tunnel is lined by the polar residues Thr682 ${ }^{\mathrm{MrpA}}$, Asp678 ${ }^{\mathrm{MrpA}}$, Asn766 ${ }^{\mathrm{MrpA}}$ as well as water W38 to W44 and exits to the periplasm close to His34 $4^{\mathrm{MrpC}}$ and Asp121 ${ }^{\mathrm{MrpC}}$. (C) Detailed of the putative periplasmic sodium exit channel as seen from MrpB. Strictly conserved Glu780 ${ }^{\mathrm{MrpA}}$ is in close proximity to the tunnel and Phe $41^{\mathrm{MrpB}}$; in the $A$ conformation Phe $41^{\mathrm{MrpB}}$ blocks the tunnel. Highly conserved regions surrounding the putative sodium exit tunnel (MrpA 676-688, MrpA 763-780, MrpB 33-45 and MrpC 66-82, compare Fig. S3) are highlighted.

With a probe radius of $1.2 \AA$ we identified a putative sodium-exit tunnel originating above the strictly conserved Phe $41^{\mathrm{MrpB}}$ that is separated from Glu687 ${ }^{\mathrm{MrpA}}$ by the hydrophobic

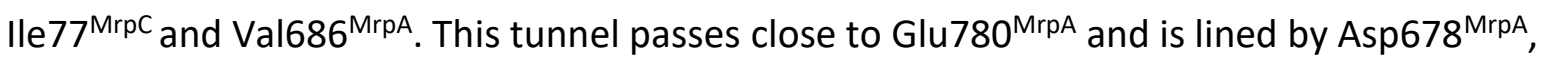
Thr682 ${ }^{\mathrm{MrpA}}$ and Asn766 ${ }^{\mathrm{MrpA}}$. In the area of the tunnel exit are the residues His34 ${ }^{\mathrm{MrpB}}$, Asp121 $1^{\mathrm{MrpB}}$ and Asp771 ${ }^{\mathrm{MrpA}}$ and water molecules W38 - W44 (Fig. 2 and Fig. 3). In agreement with a proposed function in sodium transfer all aforementioned residues are highly or strictly conserved in Mrp antiporters (Fig. S3, Table S4). Strikingly, the tunnel is blocked by one of the two alternative conformations of Phe41 ${ }^{\mathrm{MrpB}}$ (Fig. 3C, Fig. S2), suggesting a gating function for this residue, as discussed below. 


\section{Atomistic molecular dynamics simulations of the Mrp complex}

In order to obtain further dynamic insights, we performed fully atomistic MD simulations of the Mrp antiporter in several different states (see computational methods). In the S system, all amino acids were kept in their standard charged states, whereas in the P system, sidechain protonation was determined by pKa calculations prior to MD simulation runs (Tables S5 and S6). The model systems, which are based on high-resolution structural data, show high stability during MD simulations with root mean square deviation (RMSD) plateauing in the 2-3 Å range (Fig. S8B, C).

\section{Hydration of the Mrp complex}

Our MD simulation data show good agreement between the structural water content and the hydration observed in simulation trajectories. In both the S and P systems, we observed an increase in the number of internal water molecules in comparison to the structural water content (Fig. 4B). However, the number of internal water molecules plateaus in both systems, indicating that a stable hydration is reached on these simulation time scales. Remarkably, the simulation-based hydration in P states is found to be closer to the structural water content (Fig. 4B). This suggests that pKa calculations and subsequent MD simulations closely capture the charge and hydration state of the protein. Overall, the hydrated regions overlap very well with structural hydration, especially in the P state simulations (Fig. 4A).

MrpA and MrpD, which are homologous to complex I antiporter-like subunits (ND2/4/5), have both been suggested to perform proton transfer reactions (14). Analysis of MD simulation data show a hydrated connection forms towards the periplasmic side of the membrane in the MrpA subunit with high similarity to a hydrated path observed in complex I subunit ND5 (Fig. 4) $(22,27)$. Interestingly, in both ND5 and MrpA, titratable residues are conserved (Fig. S3) in this domain, and also the distortion of the planar lipid membrane is seen at the proton uptake site (exit route in complex I (22)) (Fig. S8D). In contrast to this, a similar connection to the periplasmic side is not observed in MrpD, in part due to the blockage created by hydrophobic residues (e.g. Phe393 ${ }^{\mathrm{MrpD}}$ ). This is in agreement with recent high-resolution complex I structures $(21,22)$. However, at this stage it cannot be 
excluded with certainty that a periplasmic proton transfer route opens in the MrpD subunit with some large-scale conformational changes.

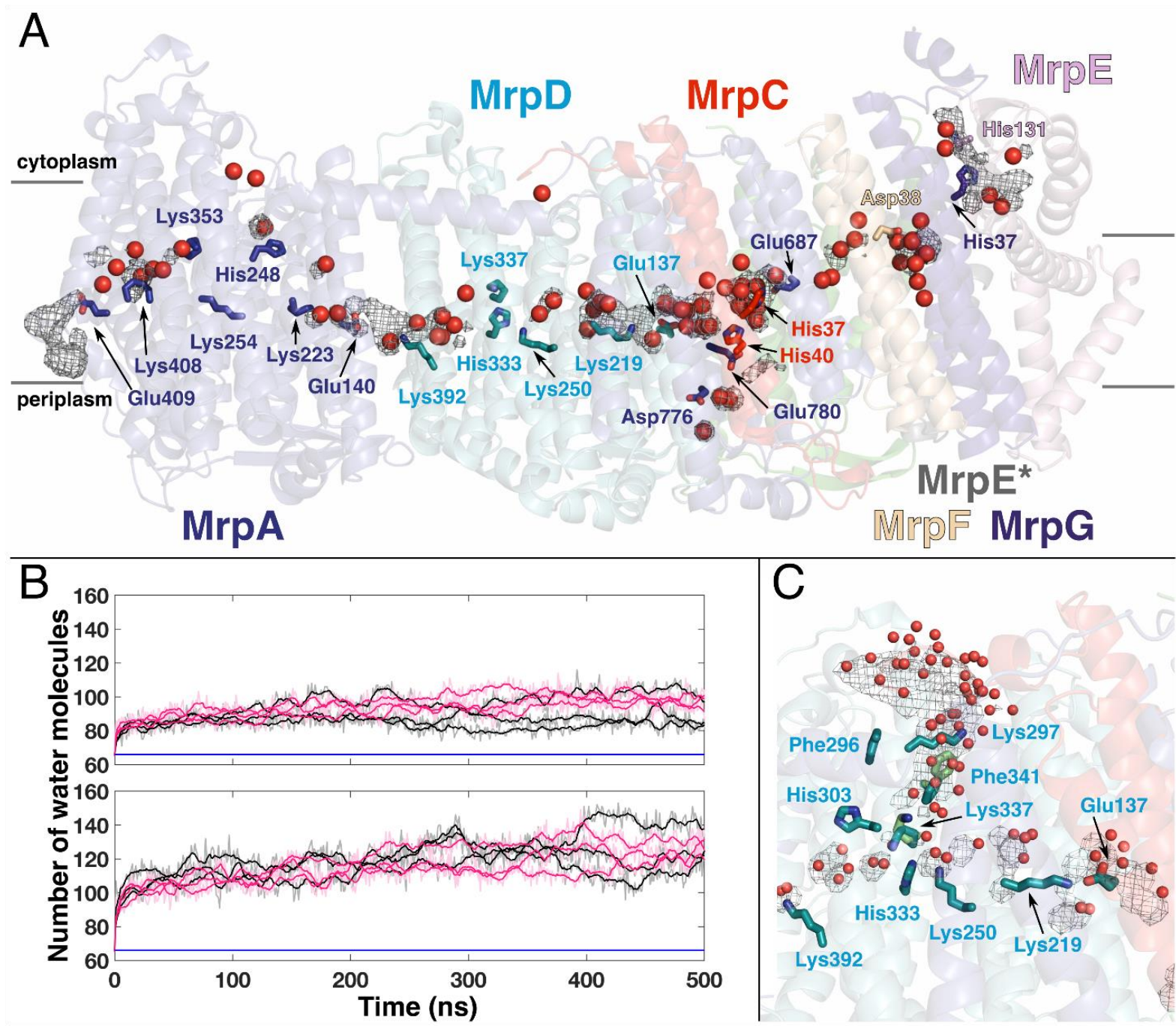

Fig. 4. Hydration of Mrp antiporter complex. (A) Overall internal hydration in simulation state $P$, where protonation state of amino acids is based on pKa calculation, is shown as water occupancy map calculated from all PB1 simulation replicas (dark grey mesh at $20 \%$ isovalue). Internal structural waters (compare Fig. 2) are shown as red spheres. Water occupancy was calculated by selecting waters within $6 \AA$ of residues displayed. (B) Number of internal water molecules in $\mathrm{P}$ (top panel) and $\mathrm{S}$ (bottom panel) simulation states. Only waters within $6 \AA$ of residues displayed in panel $A$ are counted. Black/pink traces refer to simulations started from $A / B$ alternative conformations observed in the cryoEM structure. Blue horizontal line refers to structural water content (based on water selection criterion above). (C) Hydration of MrpD subunit in simulation state $S$, where all amino acids are in their charged state. Transient water-protein based connectivity forms from cytoplasmic side to the middle of the protein. The hydration of the region occurs with the movement of Phe341 ${ }^{\mathrm{MrpD}}$ and Lys337 ${ }^{\mathrm{MrpD}}$ (cyan - structure, green - simulation). Water occupancy (calculated within $6 \AA$ of residues displayed) is shown as a grey mesh at $20 \%$ isovalue. Water molecules in red spheres are based on simulation snapshot. 
A potential connectivity towards the cytoplasmic side of the membrane is seen in the fourteen-helix bundle of MrpA in both the high-resolution cryoEM structure of Mrp complex and its MD simulations (Fig. 2 and Fig. 4). However, a similar pathway is not observed in the MrpD subunit due to the local sequence and structural variations (27) and also in part due to the blockage created by highly conserved Phe341 ${ }^{\mathrm{MrpD}}$ residue from TMH11 (Fig. S3, Fig. S4). The corresponding residue in antiporter-like complex I subunits has been suggested to be a potential gating residue (22). In our S state MD simulation, where all amino acids are modeled in their standard states, we find transient fluctuations in Phe341 ${ }^{\mathrm{MrpD}}$ and Lys337 ${ }^{\mathrm{MrpD}}$ sidechains, which led to water influx in this region and a connectivity between central Lys250 ${ }^{\mathrm{MrpD}}$ and the cytoplasmic side of the membrane appears (Fig. 4C). The hydration coupled to a conformational change in the region as well as the high conservation of Phe341 ${ }^{\mathrm{MrpD}}$ (Fig. S3) is consistent with its proposed function in gating proton transfer in both complex I and Mrp antiporter families.

\section{Protonation state-dependent conformational dynamics of His248 ${ }^{\text {MrpA }}$}

Our MD simulations in three different protonation states of His $248^{\mathrm{MrpA}}$ allows us to identify atomistic determinants of alternative $A / B$ conformations observed in the cryoEM structure. We find that His $248^{\mathrm{MrpA}}$ in $\mathrm{PA} 1$ and $\mathrm{PB} 1$, where this residue is in a charge-neutral state with $\delta$-nitrogen protonated, adopts conformations closer to the structural conformation $A$ (Fig. 5, supplementary movie S1), with transient hydrogen-bonding interactions to Thr306 ${ }^{\mathrm{MrpA}}$. However, the $B$ conformation in which His248 ${ }^{\mathrm{MrpA}}$ approaches Ser $146^{\mathrm{MrpA}}$ is never observed in these simulations. Therefore, we designed a new set of simulations in which His $248^{\mathrm{MrpA}}$ was modeled with its $\varepsilon$ nitrogen protonated and a total charge of zero (PBE setup Table S5). Remarkably, as revealed by these simulations, His248 ${ }^{\mathrm{MrpA}}$ now remained stable in the $B$ conformation, with a transient hydrogen bond to the sidechain of Ser $146^{\mathrm{MrpA}}$. In contrast to the predominant $A$ or $B$ conformations observed in the neutral state of histidine, simulations of doubly protonated His $248^{\mathrm{MrpA}}$ (system PBP in Table S5) show that it explores intermediate states between the $A$ and $B$ conformations (Fig 5). As observed above for internal protein hydration (Fig. 4), our P-state simulations closely capture the His248 ${ }^{\mathrm{MrpA}}$ conformational dynamics in contrast to the S-state (Fig. S9A). 


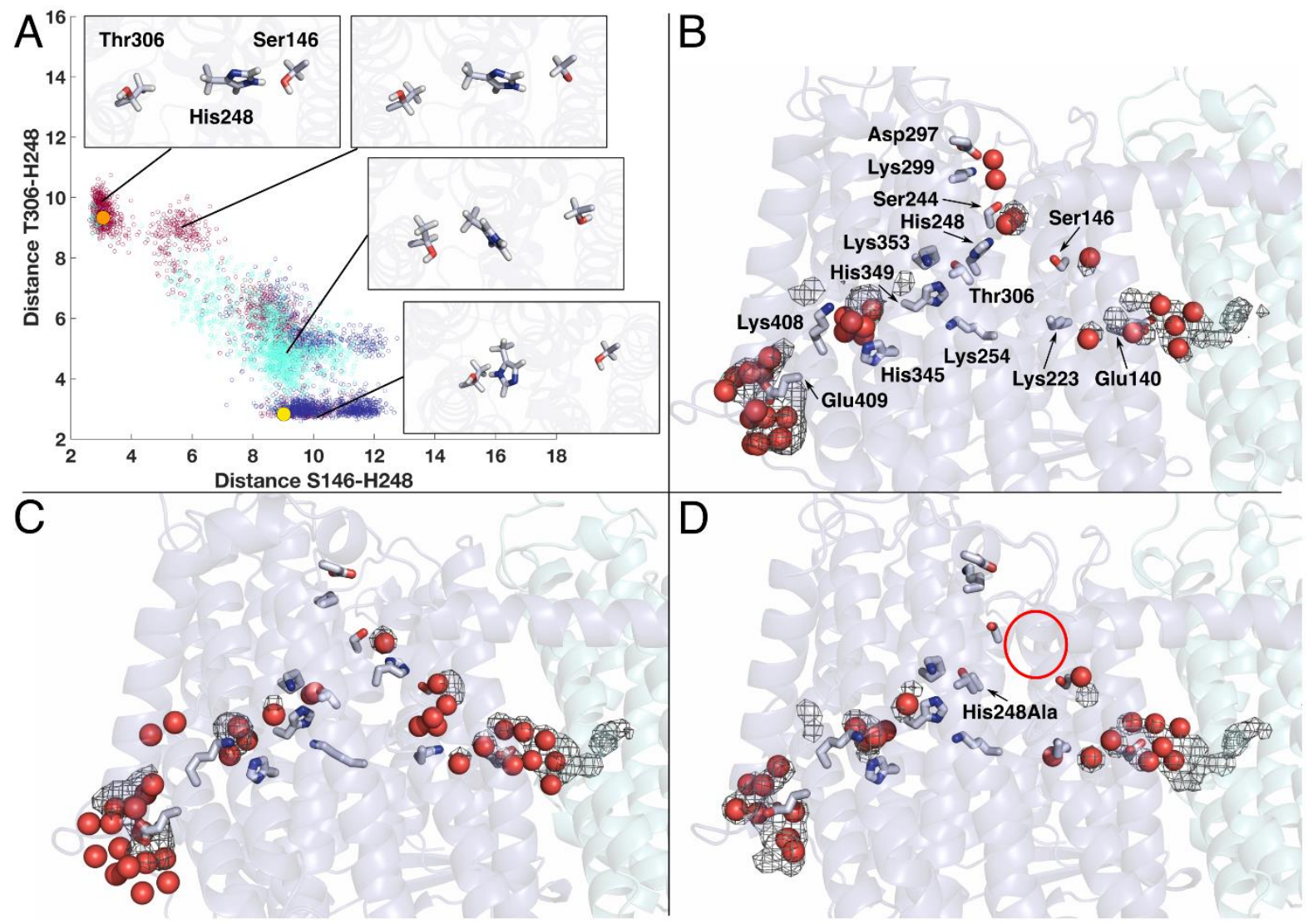

Fig. 5. Protonation-dependent conformational dynamics of His248 ${ }^{\mathrm{MrpA}}$. (A) Scatter plot displaying distances between NE2 atom of His248 ${ }^{\mathrm{MrpA}}$ and OG atoms of Ser146/Thr306 ${ }^{\mathrm{MrpA}}$ from PBE (neutral His 248 with $\varepsilon$ nitrogen protonated - burgundy), PBP (doubly protonated His248 - cyan) and PB1 (neutral His248 with $\delta$ nitrogen protonated - blue) simulations. Solid yellow and orange spheres indicate the structural distances seen in alternative $A$ and $B$ conformations (compare Fig. 2 and supplementary movie S1), respectively. Representative snapshots from different regions of the scatter plot demonstrate the conformational variety observed in the simulations. Hydration in MrpA subunit with His248 ${ }^{\mathrm{MrpA}}$ in the $A$ state from simulation PB1 (B) and in the $B$ state from simulation PBE (C). His $248^{\mathrm{MrpA}}$ in $A$ conformation next to Thr306 ${ }^{\mathrm{MrpA}}$ connects to the putative proton entry route from the periplasmic side, whereas when it changes to the $B$ conformation near Ser $146^{\mathrm{MrpA}}$, it connects to the conserved Lys/Glu pair of the MrpA subunit (Lys223 ${ }^{\mathrm{MrpA}}$ and Glu140 MrpA). His248 ${ }^{\mathrm{MrpA}}$ also maintains a cytoplasmic connectivity via a stable water molecule and strictly conserved residues Ser244 ${ }^{\mathrm{MrpA}}$ and Lys299 ${ }^{\mathrm{MrpA}}$. (D) Loss of hydration in the region (including structurally-conserved water molecule) near His248Ala ${ }^{\text {MrpA }}$ mutant (highlighted by red circle). The water occupancy map is calculated on the basis of criteria defined above for Fig. $4 \mathrm{~A} / \mathrm{B}$ and is shown at $20 \%$ isovalue. The red spheres represent instantaneous positions of water molecules from a simulation snapshot.

The protonation-dependent conformational dynamics of His248 ${ }^{\mathrm{MrpA}}$ allows it to establish a dynamic connection to the three hydrated routes described above, forming a Y-shaped junction (Fig. 5). To probe its potential role in enzyme function, we prepared the 
His248Ala ${ }^{\mathrm{MrpA}}$ mutant and found that the mutated antiporter variant was unable to sustain salt-tolerant growth of $E$. coli strain $\mathrm{KNabc}(30)$ that has no intrinsic sodium proton antiport activity (Fig. S9B). Additional MD simulations of the His248Ala ${ }^{M r p A}$ mutant showed that in the absence of the histidine sidechain, the cytoplasmic connectivity involving a structurally conserved water is lost (Fig 5D). We suggest that the loss of His248 ${ }^{\mathrm{MrpA}}$ and the associated water molecule in the His248Ala ${ }^{\mathrm{MrpA}}$ mutant disturbs the proton transfer reactions in this subunit and causes the loss of enzymatic activity.

\section{Putative sodium uptake routes}

Our MD simulations show that the sidechain of the strictly conserved Glu68 $7^{\mathrm{MrpA}}$ is mobile, and connects to three hydrated cavities; towards the Lys/Glu pair of MrpD, towards Asp38 $\mathrm{MrpF}$, and also to the cytoplasmic side (Fig. 6A). The latter cavity is consistent with our Caver analysis (Fig. 3), is primarily formed by hydrophobic residues, and hydrates rapidly when Glu687 ${ }^{\mathrm{MrpA}}$ is modeled as deprotonated (Fig. 6A). The hydrated connection forms between titratable residues at the cytoplasmic side and the Glu687 ${ }^{\mathrm{MrpA}}$ region (see also Fig. 3 and ref. (20)). The extensive conformational dynamics of Glu687 ${ }^{\mathrm{MrpA}}$ and its accessibility to three hydrated routes suggest that this residue may catalyze transfer of protons and/or sodium ions. When a sodium ion is modelled at the top of the cytoplasmic cavity near a network of charged residues, it reaches anionic Glu68 $7^{\mathrm{MrpA}}$ rapidly (within ns) and binds there (Fig. 6A). We also modeled a sodium ion near the highly conserved Aps $38^{\mathrm{MrpF}}$ and observed that it binds there in a stable manner as long as the residue is in its deprotonated state (Fig. 6B). Upon charge neutralization, Aps38 ${ }^{\mathrm{MrpF}}$ donates sodium to deprotonated Glu687 ${ }^{\mathrm{MrpA}}$ (Fig. 6C). Even though a spontaneous sodium uptake from the cytoplasmic side of the membrane was not observed, our MD simulation data suggests that there are two potential cytoplasmic sodium uptake routes (Fig. 6), and both end at the strictly conserved Glu687 MrpA. 


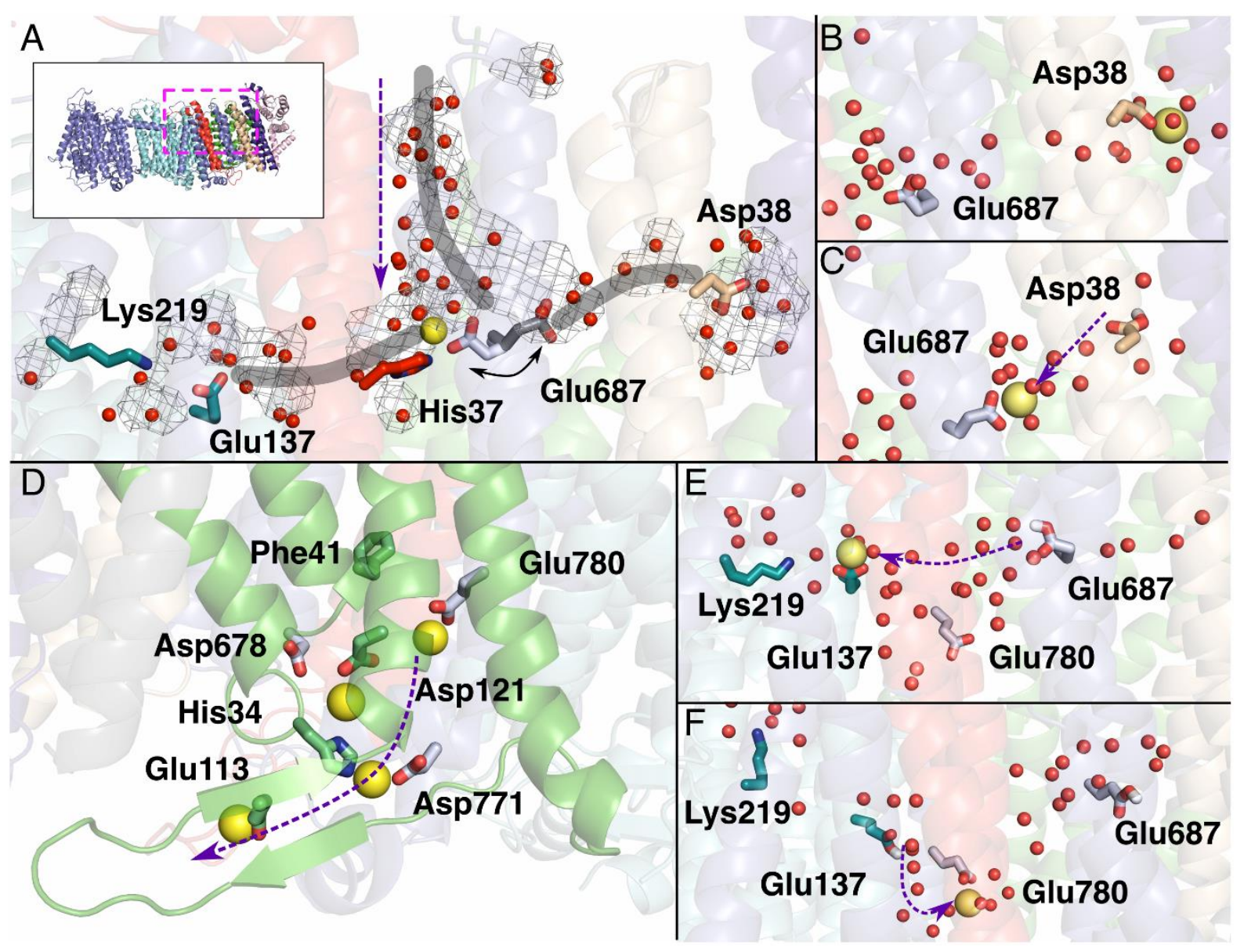

Fig. 6. MD simulations of sodium transport in Mrp antiporter. (A) The flexibility of Glu687 ${ }^{\mathrm{MrpA}}$ allows it to connect to three hydrated paths (thick grey lines) towards Lys219Glu137 pair of MrpD, towards Asp38 ${ }^{\mathrm{MrpF}}$ and towards the cytoplasmic side. The vertical purple arrow shows the direction of movement of a possible sodium ion (yellow), bound at Glu687 $\mathrm{MrpA}$, through hydrated pathway from the cytoplasmic side. (B) Sodium modeled near Asp38 ${ }^{\mathrm{MrpF}}$ binds to its sidechain, but is transferred to Glu687 ${ }^{\mathrm{MrpA}}$ upon its protonation (C). (D) Putative sodium release route with sodium ions bound to conserved residues involved in the path. (E) Protonation of Glu687 $7^{\mathrm{MrpA}}$ drives sodium to deprotonated Glu137 ${ }^{\mathrm{MrpD}}$, and upon protonation of Glu137 $7^{\mathrm{MrpD}}$ sodium is transported further to Glu780 $\mathrm{MrpA}(\mathbf{F})$ through hydrated routes.

\section{Gated sodium exit route and its protonation state dependency}

Several earlier mutagenesis studies $(29,31)$ have proposed that acidic residues are critical for potential sodium binding sites in the Mrp antiporter complex. Here, our MD simulation data reveal rapid binding (within ns) of sodium ions to residues Glu780 $\mathrm{MrpA}^{\mathrm{A}}$ and $\mathrm{Asp} 121^{\mathrm{MrpB}}$ (Fig. 6D), which are both highly conserved and have been suggested to be a part of the possible sodium exit route towards the periplasm. Additional residues that participate in 
this putative sodium exit route are Asp776 ${ }^{\mathrm{MrpA}}$, Asp678 ${ }^{\mathrm{MrpA}}$, Asp771 ${ }^{\mathrm{MrpA}}$ and His34 ${ }^{\mathrm{MrpB}}$ and Glu113 ${ }^{\mathrm{MrpB}}$, where the latter acidic residue is positioned on a $\beta$-hairpin scaffold (Fig. 6D). Our MD simulation data are also consistent with our Caver analysis and the presence of water molecules in the structure (Fig. 2, Fig. 3, Fig. S6). We observed a clear effect of the charged state of residues Glu $780^{\mathrm{MrpA}}$ and $\mathrm{Asp} 121^{\mathrm{MrpB}}$ on the binding of sodium ions. In the case when these residues are modeled charged neutral (P state runs), no sodium binding is observed, revealing that it depends on the protonation state. The binding of a sodium ion to Glu $780^{\mathrm{MrpA}}$ is found to be strongly associated with the conformation of strictly conserved Phe41 ${ }^{\mathrm{MrpB}}$, which has been observed in two alternative conformations in the cryoEM structure $(A$ and $B)$ (Fig.S2). We note that the binding of sodium to anionic Glu780 MrpA results in Phe41 ${ }^{\mathrm{MrpB}}$ turning into the alternative $A$ conformation almost immediately regardless of the starting conformation as shown in Fig. 10. However, in P state runs, where Glu $780^{\mathrm{MrpA}}$ is charge neutral, alternative conformation $B$ is also seen in which Phe $41^{\mathrm{MrpB}}$ resides next to neutral Glu780 ${ }^{\mathrm{MrpA}}$ with no sodium bound. Given the high conservancy of Phe $41^{\mathrm{MrpB}}$, these data imply an important role of this aromatic residue in coupling sodium binding to Glu780 ${ }^{\mathrm{MrpA}}$.

Our MD simulation data suggests sodium ions can travel via two potential routes from the cytoplasm and bind to the first sodium-loading site at the strictly conserved Glu687 ${ }^{\mathrm{MrpA}}$ (Fig. 6A). But they do not reveal how sodium can travel from the latter site to the second sodium loading site around Glu780 $\mathrm{MrpA}$, which sits on the exit route towards the periplasmic side (Fig. 6D). Structural and simulation analysis suggests that there are two potential routes that may allow transfer of sodium ions between the two sodium loading sites. One is a direct path that could form upon conformational changes in the highly conserved hydrophobic region comprising $1 \mathrm{le} 77^{\mathrm{MrpC}}$, Val686 ${ }^{\mathrm{MrpA}}$ and Phe41 ${ }^{\mathrm{MrpB}}$. Our Caver analysis shows that Phe41 ${ }^{\mathrm{MrpB}}$ in the alternative $B$ conformation unblocks the cavity (Fig. 3), which may allow ion transfer. Consistent with this, our MD simulations suggest that sodium binding at Glu780 MrpA triggers Phe41 ${ }^{\mathrm{MrpB}}$ in A conformation (Fig. S10), which would block the cavity. This conformational change may therefore be important for preventing back-streaming of sodium ions. A second potential route to transport sodium between the two sodium-loading sites is through the conserved acidic residue of the Lys/Glu pair of MrpD (Glu137 MrpD), which is well-connected to Glu687 ${ }^{\mathrm{MrpA}}$ (Fig. 2, 4 and 6) and is only ca. $10 \AA$ from the putative 
release site on the periplasmic side, Glu780 ${ }^{\mathrm{MrpA}}$. To track this sodium transport pathway, we performed MD simulations by varying the protonation states of amino acid residues (Glu687 $\mathrm{MrpA}$ and Asp137 $\mathrm{MrpD}$, see Table S5). We observed that the sodium ion can pass on from Glu687 ${ }^{\mathrm{MrpA}}$ to $\mathrm{Asp} 137^{\mathrm{MrpD}}$ and eventually to $\mathrm{Glu} 780^{\mathrm{MrpA}}$ in a protonation-dependent manner (Fig. 6E and 6F). Overall, our data suggests that protonation state changes coupled with conformational transitions as well as cooperative sodium binding play a key role in driving sodium translocation.

\section{Discussion}

We have determined the structure of the Mrp antiporter of $B$. pseudofirmus at $2.2 \AA$ resolution which enabled reliable modelling of 360 water molecules, of which $\sim 70$ are located in the transmembrane region. The large number of internal bound water molecules is unusual for a membrane protein complex and reminiscent of the recent high-resolution structures of respiratory complex I $(21,22)$. We find that not only titratable residues, but also many polar sidechains play a critical role in the extensive internal hydration of the antiporter complex. A substantial number of these polar residues are conserved in Mrp antiporters and respiratory complex I.

Our high-resolution cryoEM map provides evidence for dynamic processes in the catalytic cycle of the antiporter, some of which are also relevant for respiratory complex I. Of particular interest are the two clearly distinguishable conformations ( $A$ and $B$ ) of the $\operatorname{MrpA}$ TMH8 segment around His $248^{\mathrm{MrpA}}$. By switching between the $A$ and $B$ conformation, the residue bridges a gap between water-filled proton transfer pathways in the MrpA subunit. Interestingly, His $248^{\mathrm{MrpA}}$ is at the center of three possible proton transfer pathways. The alternative gating of these pathways is likely to have fundamental significance for the stoichiometry of proton and sodium transport in Mrp (Fig. 7). The preferred conformation of His $248^{\mathrm{MrpA}}$ depends not only on its protonation state but also on the location of the hydrogen atom on the imidazole moiety. The protonation-state dependent conformational dynamics of His $248^{\mathrm{MrpA}}$ and its connectivity to hydrated and conserved proton transfer pathways are in agreement with earlier MD simulations on bacterial and mitochondrial complex I $(22,27)$. Furthermore, different complex I structures also indicated two 
alternative conformations of a corresponding histidine residue (Fig. S4). Therefore, we propose that the histidine switch shown in Fig. 7 is a mechanistic feature common to Mrp antiporters and complex I.

We find that Glu687 ${ }^{\mathrm{MrpA}}$ in TMH19 of MrpA has a crucial role for sodium uptake and transfer, in agreement with previous mutagenesis data $(25,29)$. Remarkably, we observed two alternative conformations for this residue in the high-resolution cryoEM structure, which are likely to be functionally relevant. Extensive sidechain fluctuations suggested by our MD simulations are consistent with this observation. An analysis of internal cavities and MD simulation data showed that Glu68 $7^{\mathrm{MrpA}}$ is connected to three putative pathways for sodium. The first pathway connects this residue with Asp38 ${ }^{\mathrm{MrpF}}$, a conserved, functionally important residue, as indicated by site-directed mutagenesis $(14,15,29)$. Steiner and Sazanov proposed an uptake pathway for sodium that starts at the MrpE/MrpG interface and passes Asp38 $8^{\mathrm{MrpF}}$ on its way to Glu687 ${ }^{\mathrm{MrpA}}$. The second path is a more direct connection from the cytoplasm to Glu687 ${ }^{\mathrm{MrpA}}$ through a hydrophobic conduit that has some similarity with a pathway recently described for $\mathrm{MBH}(20)$ and the third path leads to the conserved Lys/Glu pair of the MrpD subunit. Our MD simulations suggest that changes in the protonation state of acidic residues (e.g. Glu687 ${ }^{\mathrm{MrpA}}$, Glu780 ${ }^{\mathrm{MrpA}}$ and Asp38 ${ }^{\mathrm{MrpF}}$ ) are central for sodium binding and transfer. Moreover, in addition to hydrated paths and sodium binding sites, our combined structural-computational data provide evidence that Phe $41^{\mathrm{MrpB}}$ might play a central role in a gating mechanism. However, it is not yet clear how sodium traverses the hydrophobic region between the two sodium-loading sites at Glu687 ${ }^{\mathrm{MrpA}}$ and Glu780 MrpA (SLS1 and SLS2 in Fig. 7). Our Caver analysis shows a tunnel from the periplasm that almost reaches Glu687 ${ }^{\mathrm{MrpA}}$ but is blocked by the hydrophobic sidechains of $11 \mathrm{e} 77^{\mathrm{MrpC}}$ and Val686 $\mathrm{MrpA}$. We speculate that a structural rearrangement might open the hydrophobic blockage, possibly also involving a rotation of TMH19. Note that the corresponding TMH3 of ND6 in complex I has been proposed to undergo a rotational motion during the transition from an $\alpha$ to a $\pi$ bulge helix $(21,32)$. MD simulations suggest a possible alternative pathway from Glu687 ${ }^{\mathrm{MrpA}}$ to the periplasm, past the Lys/Glu pair of MrpD.

Mechanistic schemes for Mrp type sodium/proton antiporters have recently been proposed by Li et al. (15) and Steiner and Sazanov (14). Several features of the model suggested by Li 
et al. are clearly at variance with our results. In particular, we consider the proposed uptake of sodium by a pathway in the MrpD subunit to be unlikely because it does not involve Glu687 ${ }^{\mathrm{MrpA}}$ and because it contains numerous positively charged residues. Although Steiner and Sazanov's model seems more consistent with our data, there are several important differences. They claim that electrostatic interactions are the main driving force in coupling sodium to proton transfer, whereas protonation or deprotonation of residues in the Cterminal domain of MrpA play no role in their model. In contrast, we propose that changes in the protonation state of e.g. Glu687 ${ }^{\mathrm{MrpA}}$ are critical for sodium binding and transfer. In fact, a direct involvement of protonation reactions would be more consistent with mechanistic principles operating in other sodium/proton antiporters. However, the exact sequence of events of proton and sodium transfer has to remain speculative at this stage. In summary, we propose a mechanism that includes the histidine switch as a previously unrecognized device for proton bifurcation in MrpA (Fig. 7). We show that Glu687 ${ }^{\mathrm{MrpA}}$ plays a central role as a loading site for sodium and propose two possible routes for sodium transfer to the cytoplasmic second sodium-loading site centered around Glu780 MrpA. The protonation state of acidic residues is closely linked to their function in binding and transfer of sodium, but further work is needed to elucidate the detailed coupling mechanism.

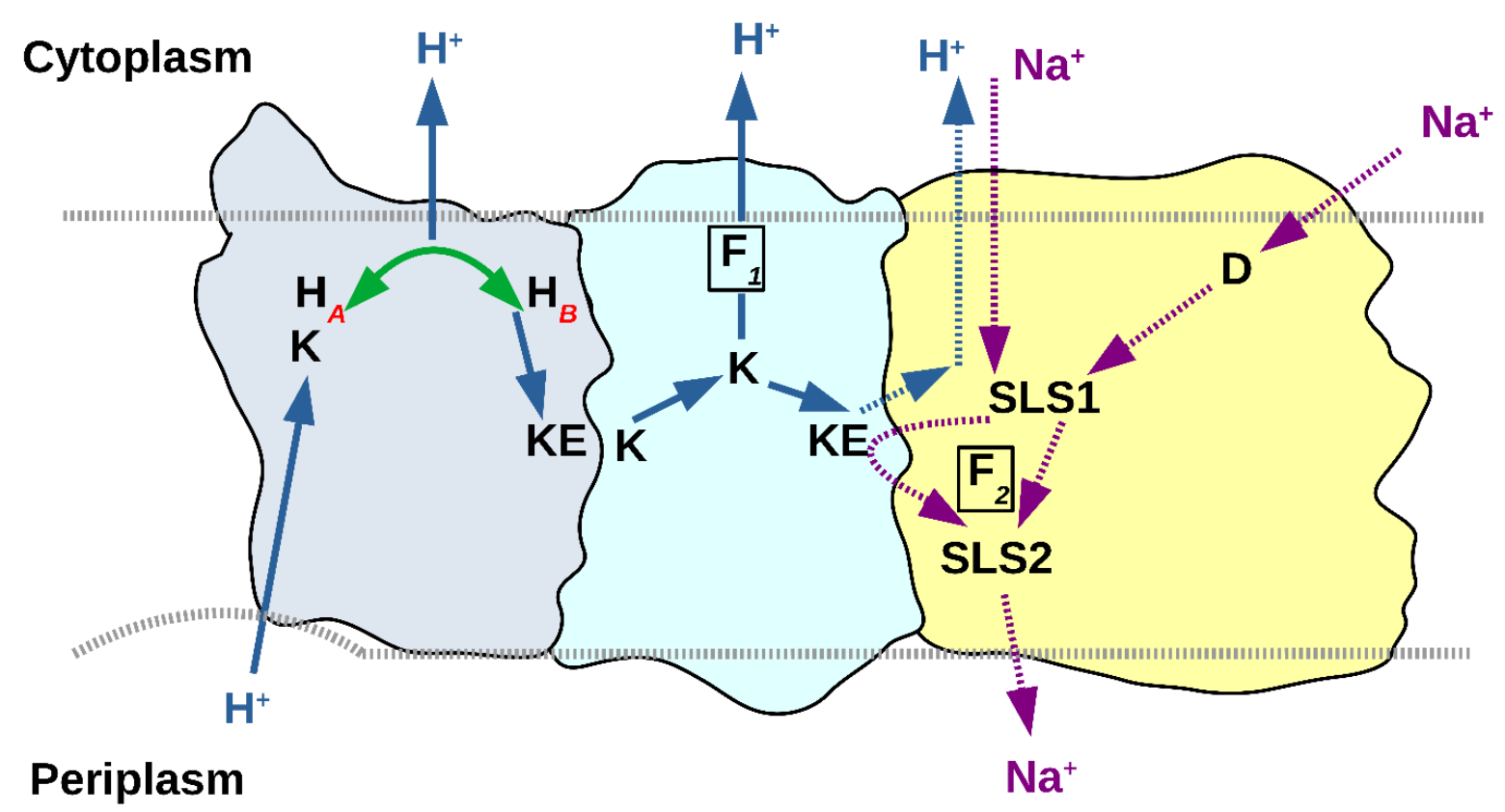


Fig.7. Proposed molecular mechanism of Mrp antiporter. Sodium ions reach the first sodium-loading site (SLS1) at the strictly conserved and conformationally mobile Glu687 ${ }^{\mathrm{MrpA}}$ via a hydrated path involving conserved $\operatorname{Asp} 38^{\mathrm{MrpF}}(\mathrm{D})$ or alternatively via a more direct but hydrophobic path observed in the structure and MD simulations (purple dotted vertical arrow). In MrpA (light blue), proton transfer occurs from the periplasm to conserved Lys353 ${ }^{\mathrm{MrpA}}(\mathrm{K})$. His248 ${ }^{\mathrm{MrpA}}$ accepts the proton from Lys353 ${ }^{\mathrm{MrpA}}$ and delivers it to the conserved Lys/Glu pair of the MrpA subunit by a histidine switch supported by our cryoEM and MD simulation data (conformational mobility of His248 ${ }^{\mathrm{MrpA}}$ is shown by green line and two alternative conformations $\mathrm{H}_{A}$ and $\mathrm{H}_{B}$ ). Transfer of a second proton to His248 ${ }^{\mathrm{MrpA}}$ (via Lys353 ${ }^{\mathrm{MrpA}}$ ) pushes the proton on the Lys/Glu pair of MrpA to Lys/Glu pair of MrpD (cyan) by electrostatic interactions. The second proton moves energetically downhill from His $248^{\mathrm{MrpA}}$ to the cytoplasm. Binding of the first proton to the Lys/Glu pair of MrpD drives the transfer of sodium bound at SLS1 (or at Glu137 ${ }^{\mathrm{MrpD}}$ ) to the second sodium-loading site (SLS2), which corresponds to the strictly conserved Glu780 $\mathrm{MrpA}$, resulting in sodium release on the periplasmic side. The proton moves to the cytoplasm either via MrpD (via the Phe-gated route, marked as $F_{1}$ ) or through the hydrophobic pathway (blue dotted vertical arrow). Our model supports a stoichiometry of $2 \mathrm{H}^{+}$per sodium translocated. Importantly, the Lys/Glu pair of MrpD is only $10 \AA$ from Glu $780^{\mathrm{MrpA}}$ (SLS2), which would result in strong electrostatic coupling. Since there are several acidic residues that can bind sodium ions, it is also possible that cooperative sodium binding plays a role in sodium translocation. Proposed proton transfer paths are shown as blue (bold and dotted) arrows and sodium translocation paths as magenta arrows. The proposed $\mathrm{Phe} 41^{\mathrm{MrpB}}$ gate is marked as $\mathrm{F}_{2}$.

\section{Materials and Methods}

\section{Expression and purification of the Bacillus pseudofirmus Mrp antiporter}

The Mrp operon from Bacillus pseudofirmus with its $170 \mathrm{bp}$ preceding sequence was amplified from the genome of $B$. pseudofirmus and subcloned into a pGEM easy vector, with the addition of a His 6 -FLAG tag at the end of MrpG subunit. We found multiple sequence discrepancies between our cloned gene and the available RefSeq genes, but all of them were either silent nucleotide substitutions or minor changes of non-conserved amino acids. The resulting plasmid was transformed into a modified $E$. coli C41 strain carrying pRARE plasmid, and protein expression was achieved by culturing the transformant in LB medium without any induction for several hours at $37^{\circ} \mathrm{C}$. The bacterial cells were harvested when protein yield saturated, which was around 3-4 hours after the culture reached OD $=0.6$. After centrifugation at 5,000 g for $12 \mathrm{~min}$, cell pellets were resuspended and lysed by sonication with a probe sonifier for $10 \mathrm{~min}$ in lysis buffer [50 mM Tris- $\mathrm{HCl}(\mathrm{pH} 8.0), 150 \mathrm{mM}$ $\mathrm{NaCl}$, and $0.1 \mathrm{mM}$ phenylmethylsulfonyl fluoride (PMSF)] on ice. Cell membranes were pelleted by ultracentrifugation at $125,000 \mathrm{~g}$ for $70 \mathrm{~min}$, homogenized in lysis buffer, frozen in liquid nitrogen, and stored at $-80^{\circ} \mathrm{C}$.

Protein purification was performed at $4^{\circ} \mathrm{C}$. Membranes were solubilized in $50 \mathrm{mM}$ Tris- $\mathrm{HCl}$ ( $\mathrm{pH}$ 8.0), $150 \mathrm{mM} \mathrm{NaCl}$, and 0.5\% lauryl maltose neopentyl glycol (LMNG) for $2 \mathrm{~h}$ and insoluble materials were removed by ultracentrifugation at $125,000 \mathrm{~g}$ for $32 \mathrm{~min}$. The supernatant was loaded onto chelating agarose resin (Qiagen) coupled with $\mathrm{Ni}$ and washed 
bioRxiv preprint doi: https://doi.org/10.1101/2022.01.24.477492; this version posted January 24, 2022. The copyright holder for this

with 5 column volumes $20 \mathrm{mM}$ Tris- $\mathrm{HCl}$ (pH 8.0), $150 \mathrm{mM} \mathrm{NaCl}, 0.05 \%$ LMNG, $1 \mathrm{mM}$ betamercaptoethanol and $20 \mathrm{mM}$ imidazole. Bound protein was eluted by increasing the imidazole concentration to $400 \mathrm{mM}$. The eluate was subjected to size exclusion chromatography on a Superose 6 Increase 3.2/300 column equilibrated in $20 \mathrm{mM}$ Tris- $\mathrm{HCl}$ (pH 8.0), $150 \mathrm{mM} \mathrm{NaCl}, 0.05 \% \mathrm{LMNG}$, and $\beta$-mercaptoethanol. The fractions containing the Mrp dimer were pooled and concentrated to $1-2 \mathrm{mg} / \mathrm{ml}$ for grid preparation.

\section{Alignments}

The Mrp operon from B. pseudofirmus was used as input for the BLAST function of UniProt (33). Each subunit was blasted separately with default conditions targeting the UniRef90 database (34) for 1000 results. Ten additional Mrp operons as well as five Complex I operons (see Figure S3) were further blasted for 100 or 250 results respectively. The results of each subunit were pooled and edited using the freeware Jalview (35). Redundant sequences with more than $90 \%$ identity and sequences whose length deviated from the mean by more than $10 \%$ were removed, resulting in 1000 to 1400 sequences for each subunit. Clustal Omega (36) was used via the JABAWS web-service (37) to perform multiple sequence alignments. The consensus histogram function with logos turned on alongside the preinstalled color scheme ClustalX (38) used with a $50 \%$ conservation threshold were utilized for visualization of conserved regions.

\section{Mutagenesis \& growth assay}

A plasmid encoding the Mrp antiporter from B. pseudofirmus carrying the point mutation $\mathrm{H} 248 \mathrm{~A}$ in MrpA was prepared by PCR-based site-directed mutagenesis. E. coli KNabc cells (30) were transformed with the plasmid and growth assays were conducted as described by Kosono et al. (31). Cells were grown for $20 \mathrm{~h}$ at $37^{\circ} \mathrm{C}$ in LBK-medium supplemented with $0-$ $1000 \mathrm{mM}$ sodium chloride using $2 \mathrm{ml}$ 96-well plates containing, mutant strain, positive and negative controls. The growth was evaluated by measuring OD600 using a SpectraMax M2/M2e Microplate Reader.

\section{Electron cryo-microscopy (cryoEM)}

Quantifoil grids ( $\mathrm{Cu} 400, \mathrm{R}$ 1.2/1.3) were pre-washed in acetone, dried, and glow-discharged twice for $45 \mathrm{sec}$ at $15 \mathrm{~mA}$ in a Pelco Glow Discharger. $3 \mu$ of purified sample were applied onto grids, blotted with a filter paper (Whatman 595 ) for $3-6$ seconds at $6^{\circ} \mathrm{C}$ and $100 \%$ humidity, and plunged into liquid ethane in a Vitrobot Mark I. For some grids, $1.5 \mathrm{mM}$ fluorinated Fos-Cholin-8 was added to the sample immediately before vitrification, which altered angular distribution of particles and improved data completeness. CryoEM images were acquired from multiple grids using EPU software on a $300 \mathrm{kV}$ Titan Krios microscope operated at $300 \mathrm{kV}$. Dose-fractionated movies were acquired at $105 \mathrm{k}$ magnification on a K3 camera, resulting in a pixel size of $0.837 \AA /$ pix. The calculated total electron exposure was $50 \mathrm{e}^{-} / \AA^{2}$ on the specimen and the exposure rate was $15 \mathrm{e}^{-} / \mathrm{pix} / \mathrm{sec}$ on the camera. CryoSPARC Live (39) was used to monitor image quality on the fly.

Single-particle data were processed in RELION 3.1 (40), including external programs such as MotionCor2 (41) for motion correction and Gctf4 (42) for contrast transfer function (CTF) 
bioRxiv preprint doi: https://doi.org/10.1101/2022.01.24.477492; this version posted January 24, 2022. The copyright holder for this preprint (which was not certified by peer review) is the author/funder, who has granted bioRxiv a license to display the preprint in perpetuity. It is made available under aCC-BY-NC-ND 4.0 International license.

estimation. Particles were first picked by a Laplacian-of-Gaussian method implemented in RELION and then by Topaz (43) and subjected to several rounds of 2D and 3D classifications to discard false positive picks. Selected particles were extracted with a box size of $512 \mathrm{px}$ and down-sampled to 360 px, resulting in a pixel size of $1.19 \AA ̊$ /pix. After 3D autorefinement with a C2 symmetry, resulting maps and poses were used to correct particle trajectories and higher-order aberrations by using Bayesian polishing and CTF refinement functions in RELION 3.1. During polishing, particles were re-extracted with a box size of 512 px and down-sampled to 400 px, resulting in a pixel size of $1.07 \AA / p i x$, which was used throughout for downstream analyses. The 3D reconstruction of these polished, higher-order aberration-corrected particles provided a consensus map at around $3 \AA$ (Figure S1A). Since this map showed blurry densities at the molecular periphery due to varying angles between the monomers, another two rounds of 3D classification were performed. The resulting more homogeneous class contained 96,337 particles, yielding a dimer map with a relatively even local resolution distribution and a global resolution of $2.96 \AA$ (Figure S1B).

To improve the map quality, we adopted a strategy similar to that used by Steiner et al. (14), whereby particles were symmetry-expanded with a C2 point group and only one monomer was covered for 3D refinement, so that signals from both monomers should align onto the same reference map. This approach improved the 3D reconstruction of the monomer significantly. After correcting for higher-order aberrations again using the pseudo-monomer map and updated particle poses, a round of 3D classification was performed without image alignment to select 513,743 symmetry-expanded particles. The final auto-refinement of these particles with local angular search (0.5 degrees) using SIDESPLITTER (44) yielded a map of the Mrp monomer with an overall resolution of $2.24 \AA$ (Figs. S1C, S2A).

\section{Model building and refinement}

An initial homology model was generated in MODELLER (45) based on the Anoxybacillus flavithermus Mrp monomer (PDB 6Z16) as a template and fitted into our 2.2-Å monomer map in ChimeraX (46). Iterative model building and refinement were performed in COOT (47) and REFMAC5 (48). The high-resolution map allowed us to fit most of the residues with defined rotamers, as clearly seen for branched-carbon amino acids such as leucine and isoleucine, or for rare backbone conformations of the cis prolines. Conversely, some residues showed ambiguous densities, which could be fitted with more than two rotameric states or even with different residue registers (Fig. S2B-E). Among these, two alternative main chain traces were possible for residues 246 - 252 in MrpA, in which His248 alternates its hydrogen-bonding partners. After modelling all the protein residues, the map clearly revealed hundreds of solvent densities to be modelled (Fig. S2F). As a guide for modelling solvent molecules, we used an Fo - Fc map calculated in REFMAC5, which took into account noise levels of two independent half-set reconstructions from RELION to calculate a weighted, sharpened map suitable for model inspection and correction. Water molecules were placed onto Fo - Fc peaks compatible with hydrogen bonding. The number of water molecules were gradually increased over several rounds of model building, refinement, and map inspection, until no significant positive peaks were left. Placed water molecules were 
validated by inspecting Fo - Fc maps after refinement so that none of them showed negative peaks. The final atomic model consists of one entire copy of MrpABCDEFG (built as chains $A-G$ ), a partial model of the adjacent MrpE subunit (chain e; only two transmembrane helices that associated tightly with MrpB were built), three lipid molecules and 360 water molecules.

\section{Computational Methods}

Atomistic molecular dynamics (MD) simulations were performed on the $2.2 \AA$-resolution structure of Mrp antiporter from Bacillus pseudofirmus. The monomeric complex was embedded in a hybrid lipid bilayer that was composed of $80 \%$ palmitoyl oleyl phosphatidylethanolamine (POPE) and 20\% palmitoyl oleoyl phosphatidylglycerol (POPG). The bilayer was obtained using CHARMM-GUI (49) and protein was inserted into the membrane with the help of OPM alignment (50). The protein-lipid system was solvated in TIP3 water molecules and $0.15 \mathrm{M} \mathrm{NaCl}$. CHARMM36 $(51,52)$ force field was used to obtain the parameters for the protein, lipids, and solvent. NBFIX (53) was applied to treat interactions between sodium ions and acidic residues. As indicated in Table S5, in setups $\mathrm{S}$ (standard), all amino acid residues were kept in their standard protonation states (e.g. all lysine, arginine, glutamate and aspartate residues were charged), whereas in setups $P$, the protonation states of titratable residues were determined by pka calculation (54) on structures in both A and B conformations (see Table S6). Table S5 also shows the starting conformation chosen for MD simulations, which were performed in both the $A$ and $B$ conformations.

The model system size was roughly 450,000 atoms, and the simulations were performed with GROMACS 2020.2 (55). The model systems were first energy minimized with the heavy atoms of protein constrained by $2000 \mathrm{~kJ} \mathrm{~mol}^{-1} \mathrm{~nm}^{-2}$ harmonic constraints. Extending the constraints to the phosphorus atoms of the lipids along the membrane normal direction, the temperature was equilibrated to $310 \mathrm{~K}$ in NVT ensemble using V-rescale thermostat (56) during a 100 ps simulation. After removing the constraints on the lipid phosphorus atoms, the systems were equilibrated in NPT ensemble using V-rescale thermostat and Berendsen barostat (57) for $1 \mathrm{~ns}$, followed by removal of all constraints and further NPT equilibration for $10 \mathrm{~ns}$. In the production phase, no constraints were applied, and the temperature and pressure were kept at $310 \mathrm{~K}$ and 1 atm using the Nose-Hoover thermostat $(58,59)$ and Parrinello-Rahman barostat (60). The timestep of the simulations was $2 \mathrm{fs}$ which was achieved using the LINCS algorithm (61). The particle-mesh Ewald method (62) was used to handle electrostatic interactions with a $12 \AA$ cutoff. The van der Waals cutoff was also $12 \AA$ with switching distance at $10 \AA$. Simulations were analysed and visualised using software VMD (63) and Pymol (64). Searches for tunnels and cavities were performed using the plugin CAVER 3.0.3 (28) in Pymol (64). The minimum probe radius was adjusted in a range of 0.7 to $1.2 \AA$ and the maximum distance of the starting point was set to $6 \AA$ otherwise default configurations were used. 


\section{Acknowledgments}

We thank the Electron Microscopy Facility of Max Planck Institute of Biophysics for cryoEM infrastructure and technical support; Sabine Häder and Heidi Betz for help in lab experiments; Juan Castillo, Özkan Yildiz, the Central IT team and the Max Planck Computing and Data Facility for support in cryoEM data processing.

We thank the Center for Scientific Computing (CSC, Finland) for extensive computational resources.

YL was supported by Toyobo Biotechnology Foundation Fellowship and Human Frontier Science Program Long-Term Fellowship.

\section{Funding:}

\section{Max Planck Society}

German Research Foundation (Deutsche Forschungsgemeinschaft) grant ZI 552/5-1 (VZ)

Sigrid Jusélius Foundation senior researcher and project grant (VS)

Jane and Aatos Erkko Foundation project grant (VS)

Academy of Finland grants 294652 and 338176 (VS)

University of Helsinki grant (VS)

Magnus Ehrnrooth Foundation grant (VS)

\section{Author contributions:}

YL expressed and purified the protein, prepared cryoEM grids, acquired and processed cryoEM data, analyzed data, drew figures and contributed to writing the manuscript. $\mathrm{OH}$ performed MD simulations, analyzed data, drew figures and contributed to writing the manuscript. AA prepared and characterized the mutant, analyzed data, drew figures and contributed to writing the manuscript. WK provided cryoEM infrastructure, supervised the cryoEM work and contributed to writing the manuscript. VS analyzed data and interpreted its mechanistic implications, supervised modeling and simulation work, drew figures and contributed to writing the manuscript. VZ initiated the project, interpreted the mechanistic implications of the structures, drew figures, and contributed to writing the manuscript.

Competing interests: Authors declare that they have no competing interests.

\section{Data and materials availability:}

All data are available in the main text or the supplementary materials. The cryoEM map has been deposited in the electron microscopy data bank (EMDB). The atomic models have 
been deposited in the protein data bank (PDB). These data will be made available as soon as possible.

\section{References}

1. M. Ito, M. Morino, T. A. Krulwich, Mrp Antiporters Have Important Roles in Diverse Bacteria and Archaea. Front Microbiol 8, 2325 (2017).

2. S. F. Pedersen, L. Counillon, The SLC9A-C Mammalian $\mathrm{Na}^{+} / \mathrm{H}^{+}$Exchanger Family: Molecules, Mechanisms, and Physiology. Physiol Rev 99, 2015-2113 (2019).

3. T. A. Krulwich, G. Sachs, E. Padan, Molecular aspects of bacterial pH sensing and homeostasis. Nat Rev Microbiol 9, 330-343 (2011).

4. E. Padan, Functional and structural dynamics of $\mathrm{NhaA}$, a prototype for $\mathrm{Na}^{+}$and $\mathrm{H}^{+}$ antiporters, which are responsible for $\mathrm{Na}^{+}$and $\mathrm{H}^{+}$homeostasis in cells. Biochim Biophys Acta 1837, 1047-1062 (2014).

5. H. Yu, G. J. Schut, D. K. Haja, M. W. W. Adams, H. Li, Evolution of complex I-like respiratory complexes. J Biol Chem, 100740 (2021).

6. G. J. Schut et al., The role of geochemistry and energetics in the evolution of modern respiratory complexes from a proton-reducing ancestor. Biochim Biophys Acta 1857, 958-970 (2016).

7. U. Brandt, Adaptations of an ancient modular machine. Science 363, 230-231 (2019).

8. K. Parey, C. Wirth, J. Vonck, V. Zickermann, Respiratory complex I - structure, mechanism and evolution. Curr Opin Struct Biol 63, 1-9 (2020).

9. V. K. Moparthi, C. Hägerhäll, The evolution of respiratory chain complex I from a smaller last common ancestor consisting of 11 protein subunits. J Mol Evol 72, 484497 (2011).

10. T. Hamamoto et al., Characterization of a gene responsible for the $\mathrm{Na}^{+} / \mathrm{H}^{+}$antiporter system of alkalophilic Bacillus species strain C-125. Mol Microbiol 14, 939-946 (1994).

11. T. Kudo, M. Hino, M. Kitada, K. Horikoshi, DNA sequences required for the alkalophily of Bacillus sp. strain C-125 are located close together on its chromosomal DNA. J Bacteriol 172, 7282-7283 (1990).

12. T. H. Swartz, S. Ikewada, O. Ishikawa, M. Ito, T. A. Krulwich, The Mrp system: a giant among monovalent cation/proton antiporters? Extremophiles 9, 345-354 (2005).

13. M. Morino, T. Suzuki, M. Ito, T. A. Krulwich, Purification and functional reconstitution of a seven-subunit mrp-type $\mathrm{Na}^{+} / \mathrm{H}^{+}$antiporter. J Bacteriol 196, 28-35 (2014).

14. J. Steiner, L. Sazanov, Structure and mechanism of the Mrp complex, an ancient cation/proton antiporter. Elife 9, (2020).

15. B. Li et al., Structure of the Dietzia Mrp complex reveals molecular mechanism of this giant bacterial sodium proton pump. Proc Natl Acad Sci U S A 117, 31166-31176 (2020).

16. C. Mathiesen, C. Hägerhäll, Transmembrane topology of the NuoL, $M$ and $N$ subunits of $\mathrm{NADH}$ :quinone oxidoreductase and their homologues among membrane-bound hydrogenases and bona fide antiporters. Biochim.Biophys.Acta 1556, 121-132 (2002).

17. E. Virzintiene et al., Structure and function of the C-terminal domain of MrpA in the Bacillus subtilis Mrp-antiporter complex--the evolutionary progenitor of the long horizontal helix in complex I. FEBS Lett 587, 3341-3347 (2013). 
18. H. Yu et al., Structure of an Ancient Respiratory System. Cell 173, 1636-1649 (2018).

19. H. Yu et al., Structure of the respiratory MBS complex reveals iron-sulfur cluster catalyzed sulfane sulfur reduction in ancient life. Nat Commun 11, 5953 (2020).

20. M. E. Mühlbauer, A. P. Gamiz-Hernandez, V. R. I. Kaila, Functional Dynamics of an Ancient Membrane-Bound Hydrogenase. J Am Chem Soc, (2021).

21. D. Kampjut, L. A. Sazanov, The coupling mechanism of mammalian respiratory complex I. Science 370, (2020).

22. K. Parey et al., High-resolution structure and dynamics of mitochondrial complex IInsights into the proton pumping mechanism. Sci Adv 7, eabj3221 (2021).

23. M. Morino et al., Single site mutations in the hetero-oligomeric Mrp antiporter from alkaliphilic Bacillus pseudofirmus OF4 that affect $\mathrm{Na}^{+} / \mathrm{H}^{+}$antiport activity, sodium exclusion, individual Mrp protein levels, or Mrp complex formation. J Biol Chem 285, 30942-30950 (2010).

24. M. Morino, S. Natsui, T. H. Swartz, T. A. Krulwich, M. Ito, Single gene deletions of mrpA to mrpG and mrpE point mutations affect activity of the $\mathrm{Mrp} \mathrm{Na}^{+} / \mathrm{H}^{+}$antiporter of alkaliphilic Bacillus and formation of hetero-oligomeric Mrp complexes. J Bacteriol 190, 4162-4172 (2008).

25. M. Morino, S. Ogoda, T. A. Krulwich, M. Ito, Differences in the phenotypic effects of mutations in homologous MrpA and MrpD subunits of the multi-subunit Mrp-type $\mathrm{Na}^{+} / \mathrm{H}^{+}$antiporter. Extremophiles 21, 51-64 (2017).

26. C. Mathiesen, C. Hägerhäll, The 'antiporter module' of respiratory chain complex I includes the MrpC/NuoK subunit -- a revision of the modular evolution scheme. FEBS Lett. 549, 7-13 (2003).

27. A. Djurabekova, O. Haapanen, V. Sharma, Proton motive function of the terminal antiporter-like subunit in respiratory complex I. Biochim Biophys Acta Bioenerg 1861, 148185 (2020).

28. E. Chovancova et al., CAVER 3.0: a tool for the analysis of transport pathways in dynamic protein structures. PLoS Comput Biol 8, e1002708 (2012).

29. Y. Kajiyama, M. Otagiri, J. Sekiguchi, T. Kudo, S. Kosono, The MrpA, MrpB and MrpD subunits of the Mrp antiporter complex in Bacillus subtilis contain membraneembedded and essential acidic residues. Microbiology 155, 2137-2147 (2009).

30. K. Nozaki, K. Inaba, T. Kuroda, M. Tsuda, T. Tsuchiya, Cloning and sequencing of the gene for $\mathrm{Na}^{+} / \mathrm{H}^{+}$antiporter of Vibrio parahaemolyticus. Biochem Biophys Res Commun 222, 774-779 (1996).

31. S. Kosono et al., Functional involvement of membrane-embedded and conserved acidic residues in the ShaA subunit of the multigene-encoded $\mathrm{Na}+\mathrm{H}+$ antiporter in Bacillus subtilis. Biochim Biophys Acta 1758, 627-635 (2006).

32. A. A. Agip et al., Cryo-EM structures of complex I from mouse heart mitochondria in two biochemically defined states. Nat Struct Mol Biol 25, 548-556 (2018).

33. U. Consortium, UniProt: the universal protein knowledgebase in 2021. Nucleic Acids Res 49, D480-D489 (2021).

34. B. E. Suzek et al., UniRef clusters: a comprehensive and scalable alternative for improving sequence similarity searches. Bioinformatics 31, 926-932 (2015).

35. A. M. Waterhouse, J. B. Procter, D. M. Martin, M. Clamp, G. J. Barton, Jalview Version 2--a multiple sequence alignment editor and analysis workbench. Bioinformatics 25, 1189-1191 (2009). 
36. F. Sievers et al., Fast, scalable generation of high-quality protein multiple sequence alignments using Clustal Omega. Mol Syst Biol 7, 539 (2011).

37. P. V. Troshin et al., JABAWS 2.2 distributed web services for Bioinformatics: protein disorder, conservation and RNA secondary structure. Bioinformatics 34, 1939-1940 (2018).

38. M. A. Larkin et al., Clustal W and Clustal X version 2.0. Bioinformatics 23, 2947-2948 (2007).

39. A. Punjani, J. L. Rubinstein, D. J. Fleet, M. A. Brubaker, cryoSPARC: algorithms for rapid unsupervised cryo-EM structure determination. Nat Methods 14, 290-296 (2017).

40. J. Zivanov et al., New tools for automated high-resolution cryo-EM structure determination in RELION-3. Elife 7, (2018).

41. S. Q. Zheng et al., MotionCor2: anisotropic correction of beam-induced motion for improved cryo-electron microscopy. Nat Methods 14, 331-332 (2017).

42. K. Zhang, Gctf: Real-time CTF determination and correction. J Struct Biol 193, 1-12 (2016).

43. T. Bepler et al., Positive-unlabeled convolutional neural networks for particle picking in cryo-electron micrographs. Nat Methods 16, 1153-1160 (2019).

44. K. Ramlaul, C. M. Palmer, T. Nakane, C. H. S. Aylett, Mitigating local over-fitting during single particle reconstruction with SIDESPLITTER. J Struct Biol 211, 107545 (2020).

45. A. Sali, T. L. Blundell, Comparative protein modelling by satisfaction of spatial restraints. J Mol Biol 234, 779-815 (1993).

46. T. D. Goddard et al., UCSF ChimeraX: Meeting modern challenges in visualization and analysis. Protein Sci 27, 14-25 (2018).

47. P. Emsley, B. Lohkamp, W. G. Scott, K. Cowtan, Features and development of Coot. Acta Crystallogr D Biol Crystallogr 66, 486-501 (2010).

48. A. Brown et al., Tools for macromolecular model building and refinement into electron cryo-microscopy reconstructions. Acta Crystallogr D Biol Crystallogr 71, 136153 (2015).

49. S. Jo, T. Kim, V. G. Iyer, W. Im, CHARMM-GUI: a web-based graphical user interface for CHARMM. Journal of computational chemistry 29, 1859-1865 (2008).

50. M. A. Lomize, I. D. Pogozheva, H. Joo, H. I. Mosberg, A. L. Lomize, OPM database and PPM web server: resources for positioning of proteins in membranes. Nucleic Acids Res. 40, D370-D376 (2012).

51. J. B. Klauda et al., Update of the CHARMM all-atom additive force field for lipids: validation on six lipid types. J Phys Chem 114, 7830-7843 (2010).

52. R. B. Best et al., Optimization of the additive CHARMM all-atom protein force field targeting improved sampling of the backbone phi, psi and side-chain chi(1) and chi(2) dihedral angles. J Chem Theory Comput 8, 3257-3273 (2012).

53. J. Yoo, A. Aksimentiev, New tricks for old dogs: improving the accuracy of biomolecular force fields by pair-specific corrections to non-bonded interactions. Phys Chem Chem Phys 20, 8432-8449 (2018).

54. M. H. Olsson, C. R. Sondergaard, M. Rostkowski, J. H. Jensen, PROPKA3: Consistent Treatment of Internal and Surface Residues in Empirical pKa Predictions. J Chem Theory Comput 7, 525-537 (2011). 
55. M. J. Abraham et al., GROMACS: High performance molecular simulations through multi-level parallelism from laptops to supercomputers. SoftwareX 1, 19-25 (2015).

56. G. D. Bussi, D.; Parrinello, M. , Canonical sampling through velocity rescaling. J. Chem. Phys. 126, (2007).

57. H. J. C. P. Berendsen, J. P. M.; van Gunsteren, W. F.; DiNola, A.; Haak, J. R. , Molecular-Dynamics with Coupling to an External Bath. J Phys Chem 81, 3684-3690 (1984).

58. S. Nosé, A unified formulation of the constant temperature molecular-dynamics methods. J Phys Chem 81, 511-519 (1984).

59. W. G. Hoover, Canonical dynamics: Equilibrium phase-space distributions. Phys.Rev.A Gen.Phys. 31, 1695-1697 (1985).

60. M. Parrinello, A. Rahman, Polymorphic transitions in single crystals: A new molecular dynamics method. J Appl Phys 52, $7182-7190$ (1981).

61. B. Hess, P-LINCS: A Parallel Linear Constraint Solver for Molecular Simulation. J Chem Theor Comput 4, 116-122 (2008).

62. T. Darden, D. York, L. Pedersen, Particle Mesh Ewald - an N.Log(N) Method for Ewald Sums in Large Systems. J Chem Phys 98, 10089-10092 (1993).

63. W. D. Humphrey, A.; Schulten, K. , VMD: Visual molecular dynamics. Journal of Molecular Graphics and Modelling 14, 33-38 (1996).

64. L. Schrödinger, W. DeLano, Pymol. Retrieved from http://www.pymol.org/pymol, (2020).

65. E. Sperling, K. Gorecki, T. Drakenberg, C. Hagerhall, Functional Differentiation of Antiporter-Like Polypeptides in Complex I; a Site-Directed Mutagenesis Study of Residues Conserved in MrpA and NuoL but Not in MrpD, NuoM, and NuoN. PLoS One 11, e0158972 (2016).

66. N. Xu et al., The Lysine 299 Residue Endows the Multisubunit Mrp1 Antiporter with Dominant Roles in $\mathrm{Na}^{+}$Resistance and pH Homeostasis in Corynebacterium glutamicum. Appl Environ Microbiol 84, (2018). 